\title{
RELEVANCE OF WHOLE BODY VIBRATION EXERCISE IN SPORT: A SHORT REVIEW WITH SOCCER, DIVER AND COMBAT SPORT
}

\section{Danielle Soares Morel*1,3, Carla da Fontoura Dionello ${ }^{1,3}$, Eloá Moreira-Marconi ${ }^{2,3}$, Samuel Brandão- Sobrinho-Neto ${ }^{3,4}$, Laisa Liane Paineiras-Domingos ${ }^{1,3}$, Patrícia Lopes Souza ${ }^{1,3}$, Danúbia da Cunha Sá- Caputo $^{1,3}$, Glenda Dias ${ }^{3}$, Claudia Figueiredo ${ }^{3,4}$, Roberto Carlos Resende Carmo ${ }^{3}$, Patrícia de Castro Paiva $^{3,4}$, Cintia Renata Sousa-Gonçalves ${ }^{1,3}$, Cristiane Ribeiro Kütter ${ }^{3,4}$, Eliane de Oliveira Guedes- Aguiar $^{3}$, Ross Cloak $^{5}$, Mario Bernardo-Filho ${ }^{3}$}

${ }^{1}$ Universidade do Estado do Rio de Janeiro, Programa de Pós-Graduação em Ciências Médicas, Rio de Janeiro, Brazil. ${ }^{2}$ Universidade do Estado do Rio de Janeiro, Programa de Pós-Graduação em Fisiopatologia Clínica e Experimental, Rio de Janeiro, Brazil. ${ }^{3}$ Universidade do Estado do Rio de Janeiro, Instituto Biologia Roberto Alcântara Gomes, Departamento de Biofísica e Biometria. ${ }^{4}$ Universidade do Estado do Rio de Janeiro, Mestrado Profissional em Saúde, Medicina Laboratorial e Tecnologia Forense, Rio de Janeiro, Brazil. ${ }^{5}$ The University of Wolverhampton, Institute of Sport, Walsall Campus, UK

*Corresponding Author E-mail: $\underline{\text { danisoaresmorel@gmail.com }}$

\begin{abstract}
Background: Whole body vibration exercise (WBVE) has been used as a safe and accessible exercise and important reviews have been published about the use of this exercise to manage diseases and to improve physical conditions of athletes The aim of this paper is to highlight the relevance of WBVE to soccer players, divers and combat athletes.

Material and methods: This study was made through a systematic review of publications involving WBVE and the selected sports in two databases (Pubmed and PEDRo).

Results: It were identified 10 studies involving WBVE and sports (6 of soccer, 2 of diving and 2 of sport combat) with 156 subjects ( 80 soccer players, 32 divers and 44 combat athletes), with age from 17 to 44 years old.

Conclusion: The use of WBVE has proven to be a safe and useful strategy to improve the physical conditions of players of different sports. These findings may have clinical relevance and should be considered as a strategy to be used to try improve the physical conditions of players.
\end{abstract}

Keywords: whole body vibration exercises, sports, soccer, combat sport, diver.

\section{Introduction}

Exercise is a planned, structured and repetitive physical activity, with specific objectives as weight loss, improvement or maintenance of one or more components of physical fitness. According to Sport Accord (2016), sport is a exercise that should have an element of competition. Physiological and technical demands of the sport have led coaches and clinicians to look for the best methods of preparation for the athletes to perform their optimum and to permit people to perform the exercises with security.

Soccer is a global sport, according to FIFA (2016), and involves, to varying degrees, kicking a ball to score a goal. Running is the predominant activity and explosive efforts during sprints, duels, jumps and changes of direction are important performance factors, requiring maximal strength and anaerobic power of the neuromuscular system (Cloak et al, 2016). These physiological responses will depend on the quality of the physical preparation of the athletes with proper exercises.

Combat sport is a competitive contact sport with one-on-one combat. Combat athletes train and compete barefoot. In combat sports such static stretching (SS) is predominantly used as part of the warm-up process. It is also used at the end of training sessions to improve and/or maintain flexibility. However, some research indicates that SS may have detrimental effects on explosive strength (i.e. vertical jump), maximum strength, speed, and agility if used before muscular activity including these biomotor abilities (Kurt, 2015). To overcome the negative effects of SS, it has been recommended to replace SS with dynamic stretching (DS) in the warm-up period. DS has no detrimental effects and may have positive effects on subsequent muscular activity (Kurt, 2015). DS is not as effective as SS at improving flexibility (Kurt, 2015).

Diving is a dynamic aquatic sport that combines skill, coordination, flexibility and muscular power of lower limbs (Dallas et al., 2015). It requires large range of motion (ROM) movements in order to execute unusual or unique body positions and skills of artistic nature (Dallas et al., 2015). Excessive muscular strength of the lower body compared to upper body in springboard divers is an obvious necessity that needs to be achieved via specific strength training (Dallas et al., 2015). Another approach related to the diving is the prevention of decompression sickness (DCS) in scuba diving. Although currently available decompression tables and algorithms are capable of reducing the risk of 19 


\section{Morel et al., Afr J Tradit Complement Altern Med., (2017) 14(S): 19-27 \\ https://doi.org/10.21010/ajtcam.v14i4S.3}

DCS, they cannot eliminate it completely (Germonpre et al., 2009). A substantial proportion of DCS cases are classified "unexplained" when the diver has complied with the procedures imposed by the chosen decompression model (dive computer or dive table). So, a large body of research is currently directed at reducing the incidence of (micro)gas bubbles during decompression. Research into the optimization of decompression procedures is hampered by the large inter- (and even intra-) individual variability of post-dive decompression bubble formation (Germonpre et al., 2009). Another approach to reducing bubble formation after a dive is to "pre-condition" the diver prior to immersion. The general idea behind this approach is that post-dive decompression bubbles originate from the endothelial surface. Reports have been published on beneficial effects of pre-dive exercise (Germonpre et al., 2009; Claybaugh et al., 2004), oxygen breathing, pre-dive hyperbaric sessions (Germonpre et al., 2009), heat preconditioning (Lee et al., 2009), hydration, and nitric oxide (NO) donor administration. These experiments try to influence bubble formation by modifying biophysical or chemical properties of the endothelial surface, on which gas bubbles or nuclei are presumed to be forming.

Among the various modalities of exercises, the scientific interest in whole body vibration exercise (WBVE) generated when mechanical vibrations are transmitted when a subject is in contact with an oscillating/vibratory platform (OVP). WBVE has been used as a safe and accessible exercise and important reviews have been published about the use of this exercise to manage diseases and to improve physical conditions of athletes (Kurt and Pekünlü, 2015). The mechanical vibration produced in an OVP is a physical agent with an oscillatory motion about an equilibrium point (Rauch et al., 2010) and can be determined by biomechanical parameters: frequency, amplitude, peak-to-peak displacement and peak acceleration. Besides them, some other parameters must also be considered, as types of OVP (synchronous, alternated or triplanar (Rittweger, 2010; Signorile, 2011), duration (working time), time of rest between bouts, periodicity of the sessions and position adopted by subject on the OVP (Rauch et al., 2010).

Some studies conducted on well-trained/elite athletes showed an acute performance-enhancing effect of WBVE on vertical jump height, mechanical power (Kurt, 2015; Wyon et al., 2010; Kurt and Pekünlü, 2015), flexibility (Osawa et al., 2013; Dallas et al., 2015) and muscular activity (Cochrane, 2011; Marín et al., 2010).

Further studies are needed to determine optimal WBVE protocols and variable combinations for elite/welltrained athletes to enhance performance (Kurt and Pekünlü, 2015). Each sport has its own specific requirements, such as training intensity/frequency/volume, diet, environmental conditions, dominant energy system, psychological factors, body dimensions, and predominantly used muscles. This information has raised important questions that thisabout the relevance of the WBVE to sports. The aim of this review is to highlight the relevance of WBVE to soccer players, divers and combat athletes.

\section{Material and Methods Search Strategy}

Three reviewers independently accessed bibliographical databases (PubMed and/or PEDRo) through Universidade do Estado do Rio de Janeiro on January $2^{\text {th }} 2016$ and all publications were initially considered up to this date. The search was performed to verify the total number of publications (NP) in these databases. The keywords were "whole body vibration" and "sports", "whole body vibration" and "soccer", "whole body vibration" and "sports" and "soccer", "whole body vibration" and "divers", "whole body vibration" and "diving", "whole body vibration" and "sports" and "divers", "whole body vibration" and "combat athletes", "whole body vibration" and "sports" and "combat athletes", "whole body vibration" and "fighting sport" and "whole body vibration" and "combat sport".

\section{Eligibility Criteria}

The inclusion criteria were: randomized controlled trials (RCTs), exercise interventions based on the use of WBVE in soccer, combat sports or divers, clearly detailed parameters of exercise containing a description of at least frequency of the vibration and written in English. Studies were excluded if they were reviews, if they didn't meet the minimum requirements regarding the description of training variables or if they were case reports. In a second round, and in the absence of RCT's, single group experimental studies were also considered (cross-over designs).

As the three reviewers carried out the searches for publications independently with the established keywords, data were independently abstracted and disagreements were discussed until a consensus was reached. Full papers were included for this narrative review if they met the search criteria and described a study using WBVE generated by an OVP used to verify the flow independently on the year of publication. 


\section{Level of evidence of the selected papers}

Included studies were classified according to the National Health and Medical Research Council hierarchy of Evidence - NHMRC, 2003-2007 (Merlin et al., 2009) (Figure 1). Each article was assigned to a one reviewer, crosschecked by a second reviewer and in case of disagreement, a third reviewer was consulted until consensus was reached.

\begin{tabular}{ll}
\hline Level of evidence & Description \\
\hline I & Systematic review of level II studies \\
II & Randomized controlled trial \\
III-1 & $\begin{array}{l}\text { Pseudo-randomized controlled trial (alternate allocation, as a crossover study or some } \\
\text { other similar method } \\
\text { Comparative study with concurrent controls (non-randomised experimental trial, cohort } \\
\text { III-2 }\end{array}$ \\
III-3 & $\begin{array}{l}\text { Comparative study without concurrent control (Historical control, two or more single arm } \\
\text { study, interrupted time series without a parallel control group }\end{array}$ \\
IV & Case series with either post-test or pre-test/post-test outcomes
\end{tabular}

*-adapted from NHMRC, 2003-2007 (Merlin et al., 2009)

Figure 1 - Designation* of levels of evidence (LE) according to National Health and Medical Research Council (NHMRC 2003-2007)

\section{Results \\ WBVE and soccer}

Six potentially relevant studies were identified. A screening of the titles excluded two articles and then the remaining four studies were analyzed concerning the eligibility criteria. Four publications with a total of 80 participants (100\% male) with age ranging from 20 up to 25 years old were analyzed.

Table I shows included studies involving WBVE and soccer with the sample size, gender and age of the participants, outcomes assessed and main findings, biomechanical parameters of the mechanical vibration and information about protocols and level of evidence. The level of evidence of the studies included in this review was III-1 according to the NHMRC in two studies (Rønnestad et al., 2012, Padulo et al., 2014), and II in another two studies (Lovell et al., 2013; Cloak et al., 2016).

Cloak et al. (2016) have used the Y balance test (YBT) scores and dynamic postural stability index (DPSI) measured pre and post and observed that DPSI was significantly lower in the elite players in acute WBVE compared to amateur players. YBT anterior reach distance showed significant improvements in both group of players due to WBVE. Rønnestad et al. (2012) assessed the 40-m sprint was performed 1 minute after the preconditioning exercise and have verified that performing the preconditioning exercise with WBVE at a frequency of $50 \mathrm{~Hz}$ resulted in a superior 40-m sprint performance compared to preconditioning exercise without WBVE. There was no difference between preconditioning exercise with WBVE at a frequency of $30 \mathrm{~Hz}$ and no-WBVE condition. Lovell et al. (2013) determined at regular intervals during fixed-intensity soccer stimulations (SAFT), vastus lateralis temperature (T(m)) was recorded, and players also performed maximal counter-movement jumps (CMJ), 10-m sprints, and knee flexion and extension contractions. Padulo et al. (2014) used Best Time (BT), worst time (WT), total time (TT), fatigue index (FI) of repeated sprint ability (RSA), and post-test blood lactate (BLa) and have found no differences between RSA1 and RSA3. Significant differences in all variables studied. TT $=$ [RSA2 $0.93 \%$ and $1.68 \%$ lower than RSA1 and RSA3 respectively; $\mathrm{p}<0.05], \mathrm{BLa}=$ [RSA2 $16.97 \%$ and $14.73 \%$ greater than RSA1 and RSA3 respectively; $\mathrm{p}<0.001], \mathrm{WT}=$ [RSA2 $1.90 \%$ and $2.93 \%$ lower than RSA1 and RSA3 respectively; $\mathrm{p}<0.01$ ], and FI $=$ [RSA2 30.64\% and 40.15\% lower than RSA1 and RSA3 respectively; $\mathrm{p}<0.0001]$. 
https://doi.org/10.21010/ajtcam.v14i4S.3

Morel et al., Afr J Tradit Complement Altern Med., (2017) 14(S): 19-27

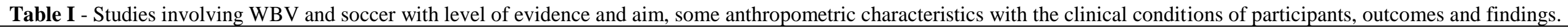

$\begin{array}{llll}\text { Table I - Studies involving WBV and soccer with level of evidence and aim, some anthropometric characteristics with the clinica } \\ \text { Parameter } & \text { Cloak et al, } 2016 & \text { Radulo et al, 2014 }\end{array}$ Lovell et al, 2013

\begin{tabular}{|c|c|}
\hline $\begin{array}{l}\text { Sample } \\
\text { Size/oender }\end{array}$ & 44 healthy male players ( 22 elite \\
\hline Age (yrs old) & $22.1 \pm 2.1$ \\
\hline Aims & $\begin{array}{l}\text { Acute effects of WBVT on } \\
\text { balance and stability amongst } \\
\text { elite and amateur soccer players. }\end{array}$ \\
\hline Outcomes & $\begin{array}{l}\text { YBT scores and DPSI measured } \\
\text { pre and post. }\end{array}$ \\
\hline Results & $\begin{array}{l}\text { DPSI was significantly lower in } \\
\text { the elite players in the acute } \\
\text { WBVT compared to amateur } \\
\text { players (F1, 40=6.80). YBT } \\
\text { anterior reach distance showed } \\
\text { an improvement in both amateur } \\
\text { and elite players in the acute } \\
\text { WBVT group (F1, } 40=32.36 \text { ). }\end{array}$ \\
\hline
\end{tabular}

17 male players

9 male amateur players

10 semi-professional male player

\section{$16.71 \pm 0.4$}

Acute effects of WBV on RSA.

$23 \pm 2$

Effect of adding WBV to body-loaded halfsquats, performed as preconditioning activity to the 40-m sprint test.

BT, WT, TT, FI of RSA, and post-test BLa.

The 40-m sprint was performed 1 minute after the preconditioning exercise.

$20 \pm 1$

Effects of WBV and a field-based re-warm-up during HT on subsequent physical performance measures during a simulated soccer game.

At regular intervals during SAFT(90), vastus lateralis temperature $(\mathrm{T}(\mathrm{m}))$ was recorded and players also performed maximal CMJ, 10-m sprints and knee flexion and extension contractions.

No differences between RSA1 and RSA3. Performing the preconditioning exercise with WBV at a frequency of $50 \mathrm{~Hz}$ resulted Significant differences in all variables studied. $\mathrm{TT}=[$ RSA $20.93 \%$ and $1.68 \%$ lower than RSA 1 and RSA3 respectively, BLa $=$ [RSA2 $16.97 \%$ and $14.73 \%$ greater than RSA1 and RSA3 respectively; $\mathrm{WT}=[\mathrm{RSA} 21.90 \%$ and $2.93 \%$ lower than RSA1 and RSA3 respectively; and $\mathrm{FI}=[\mathrm{RSA} 230.64 \%$ and $40.15 \%$ lower than RSA1 and RSA3 respectively. When comparing individual sprints, WBV showed a significant effect at the 5th sprint: RSA2 $2.29 \%$ and $2.95 \%$ lower than RSA1 and RSA3 respectively, while at the 6th sprint: RSA2 $2.75 \%$ and $4.09 \%$ lower than RSA1 and RSA3 respectively.

in a superior $40-\mathrm{m}$ sprint performance compared to preconditioning exercise without WBV $(5.48 \pm 0.19$ vs. $5.52 \pm 0.21$ seconds, respectively, There was no difference between preconditioning exercise with WBV at a frequency of $30 \mathrm{~Hz}$ and the no-WBV condition.

At the start of the second half, sprint and CM performance and eccentric hamstring peak torque were significantly reduced compared with the end of the first half in $\mathrm{CON}(\mathrm{P} \leq 0.05)$. There was no significant change in these parameters over the HT period in the $\mathrm{WBV}$ and IAE interventions $(\mathrm{P}>0.05)$. The decrease in $\mathrm{T}(\mathrm{m})$ over the HT period was significantly greater for $\mathrm{CON}$ and WBV compared with IAE $(\mathrm{P} \leq 0.01)$.

Synchronicus

PPD $2.2 \mathrm{~mm}$

Synchronicus

50 or 30

No informed

Half squat

Stood, knees flexed at $\sim 90^{\circ}$

$1 \times 20$ seconds

$15 \times 30 \mathrm{~s}$

$2 / 48 \mathrm{~h}$

III-1
III-1
Synchronicus

PPD $0.83 \mathrm{~mm}$

Partial squat posture $\left(30^{\circ}\right.$ knee flexion

$3 \times 60 \mathrm{~s}$

$3 \times 60$ s/ 60-s recovery periods.
Sets/Time
series/ rest

Level

of II
II 
When comparing individual sprints, WBVE showed a significant effect at the 5th sprint: RSA2 2.29\% and $2.95 \%$ lower than RSA1 and RSA3 respectively ( $<<0.005$ ), while at the 6th sprint: RSA2 $2.75 \%$ and $4.09 \%$ lower than RSA1 and RSA3 respectively; $\mathrm{p}<0.005$. Lovell (2013) have found that at start of second half, sprint and CMJ performance and eccentric hamstring peak torque were significantly reduced compared with the end of the first half in control - $\mathrm{CON}(\mathrm{P} \leq 0.05)$. There was no significant change in these parameters over the half-time period (HT) period in the WBVE and intermittent agility exercises interventions - IAE $(\mathrm{P}>0.05)$. The decrease in T(m) over the HT was significantly greater for CON and WBVE compared with IAE $(\mathrm{P} \leq 0.01)$. In all the studies a synchronicus platform was used and the frequency ranged from 30 up to $50 \mathrm{~Hz}$. The individuals were on the base of the platform in squat position with knees bent $100^{\circ}$ (Cloak et al., 2016), 90 (Padulo et al., 2014), 30 (Lovell et al., 2013) and Rønnestad et al. (2012) performed a half squat. Regarding the length of the protocol, only a single session was used. Working time in sessions varied from 20 up to 60 seconds.

\section{WBVE and divers} were analyzed.

Two publications with a total of 32 participants $(75 \%$ male) with age ranging from 17 up to 44 years old

Table II shows included studies about WBVE and divers with the sample size, gender and age of participants, outcomes assessed and the main findings, biomechanical parameters of the mechanical vibration and information about protocols and level of evidence. Level of evidence of both studies included were III- 1 according to NHMRC.

Dallas et al. (2015) evaluated flexibility and explosive strength of lower limbs before (Pre), immediately after (Post 1) and $15 \mathrm{~min}$ after the end of vibration exposure (Post 15). It was observed that flexibility and explosive strength of lower limbs were significantly higher in both WBVE protocols compared to no-vibration group (NVG). The greatest improvement in flexibility and explosive strength, occurred immediately after vibration treatment, was maintained 15 min later in both WBVE protocols, whereas NVG revealed a significant decrease 15 min later, in all examined strength parameters. Germonpre et al. (2009) measured the post-dive bubbles precordially 30, 60, and 90 min after the dive and were graded according to the Kissman Integrated Severity Score (KISS) protocol, with and without knee flexion. Arterial endothelial function was measured before and after vibration using flow mediated dilation (FMD) measurement. He reported a significant reduction in bubble scores was observed after the "vibration" dive. Synchronicus platform was used and the frequency ranged from 30 up to $50 \mathrm{~Hz}$. The individuals were on the base of the platform in static squat at a knee angle of $120^{\circ}$, they did dynamic squat of $2 \mathrm{~s}$ up and $2 \mathrm{~s}$ down at a knee angle ranging from $120^{\circ}$ to $180^{\circ}$ (Dallas et al., 2015) or they lay on motionless on the mattress during the entire vibration session (Germonpre et al., 2009). A single session was used in the two publications. The working time in the sessions varied from 2 up to $30 \mathrm{~min}$. 
https://doi.org/10.21010/ajtcam.v14i4S.3

Morel et al., Afr J Tradit Complement Altern Med., (2017) 14(S): 19-27

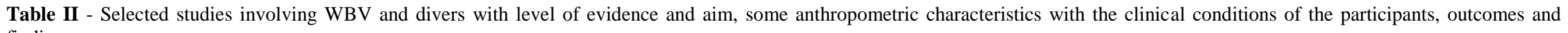
findings.

\begin{tabular}{|c|c|c|}
\hline Parameter & Dallas et al, 2015 & Germonpré et al, 2009 \\
\hline Sample & 18 competitive divers( 10 males and 8 females $)$ & 14 healthy male military divers \\
\hline \multicolumn{3}{|c|}{ 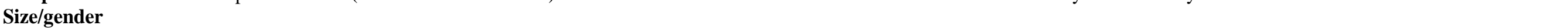 } \\
\hline Age (yrs old) & $19 \pm 2$ & $23-44$, mean 29 yrs \\
\hline Aims & $\begin{array}{l}\text { Acute effects of different vibration loads (frequency and amplitude) of WBV on flexibility and } \\
\text { explosive strength of lower limbs in springboard divers. }\end{array}$ & Effects of pre-dive WBV on post-dive bubble formation. \\
\hline Outcomes & $\begin{array}{l}\text { Flexibility and explosive strength of lower limbs were measured before (Pre), immediately after } \\
\text { (Post 1) and } 15 \mathrm{~min} \text { after the end of vibration exposure (Post 15). }\end{array}$ & $\begin{array}{l}\text { Post-dive bubbles were measured precordially } 30,60 \text {, and } 90 \text { min } \\
\text { after the dive and were graded according to KISS protocol, with and } \\
\text { without knee flexion. Arterial endothelial function was measured } \\
\text { before and after vibration using FMD measurement. }\end{array}$ \\
\hline Results & $\begin{array}{l}\text { Flexibility and explosive strength of lower limbs were higher in both WBV protocols compared to } \\
\text { NVG. The greatest improvement in flexibility and explosive strength, which occurred } \\
\text { immediately after vibration treatment, was maintained } 15 \text { min later in both WBV protocols, } \\
\text { whereas NVG revealed a significant decrease } 15 \text { min later, in all examined strength parameters. }\end{array}$ & $\begin{array}{l}\text { Significant reduction in bubble scores was observed after the } \\
\text { "vibration" dive. }\end{array}$ \\
\hline Type of OVP & Synchronicus & Synchronicus \\
\hline Frequency $(\mathbf{H z})$ & $30-50$ & $35-40$ \\
\hline $\begin{array}{l}\text { Amplitude/PPD } \\
(\mathbf{m m})\end{array}$ & $2-4$ & \\
\hline Position & $\begin{array}{l}\text { Static squat at } 120^{\circ} \text { knee flexion, dynamic squat of } 2 \mathrm{~s} \text { up and } 2 \mathrm{~s} \text { down at a knee angle ranging } \\
\text { from } 120^{\circ} \text { to } 180^{\circ}\end{array}$ & $\begin{array}{l}\text { The subject lay motionless on the mattress during the entire vibration } \\
\text { session }\end{array}$ \\
\hline Sessions & 1 & 1 \\
\hline $\begin{array}{l}\text { Sets/Time series/ } \\
\text { rest }\end{array}$ & $1 / 2 \min (4 \times 30 \mathrm{~s} / 30 \mathrm{~s}$ rest between sets $)$ & $1 / 30 \mathrm{~min}$ \\
\hline Level of evidence & III-1 & III-1 \\
\hline
\end{tabular}




\section{Morel et al., Afr J Tradit Complement Altern Med., (2017) 14(S): 19-27}

\section{https://doi.org/10.21010/ajtcam.v14i4S.3}

\section{WBVE and combat athletes}

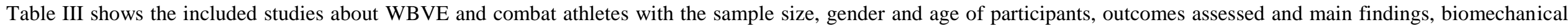
parameters of the mechanical vibration and information about protocols and level of evidence. The level of evidence of both studies included were III-1 according to the NHMRC.

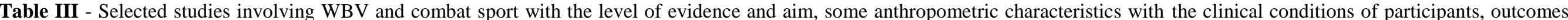
and findings.

\begin{tabular}{|c|c|c|}
\hline Parameter & Kurt and Pekünlü, 2015 & Kurt , 2015 \\
\hline Sample & 20 athletes (12 females and 8 males) & 24 well-trained male athletes \\
\hline \multicolumn{3}{|l|}{ Size/gender } \\
\hline Age (yrs old) & $22.8 \pm 3.1$ & $22.7 \pm 3.3$ \\
\hline Aims & $\begin{array}{l}\text { Effect of WBV training on maximal strength, squat jump, and flexibility } \\
\text { of well-trained combat athletes. }\end{array}$ & $\begin{array}{l}\text { Effect of local vibration and WBV on lower body flexibility and to assess whether vibration } \\
\text { treatments were more effective than traditionally used SS and DS methods }\end{array}$ \\
\hline Outcomes & $\begin{array}{l}\text { Handgrip, squat jump, trunk flexion and isometric leg strength tests were } \\
\text { performed after each intervention. }\end{array}$ & $\begin{array}{l}\text { SS and DS in } 4 \text { sessions of equal duration } 48 \text { hours apart in a randomized balanced order. } \\
\text { During a } 15 \text {-minute recovery after each protocol, subjects performed S\&Rtest. }\end{array}$ \\
\hline Type of OVP & Synchronicus & Synchronicus \\
\hline Frequency (Hz) & 26 & 30 \\
\hline $\begin{array}{l}\text { Amplitude/PPD } \\
(\mathbf{m m})\end{array}$ & ( & 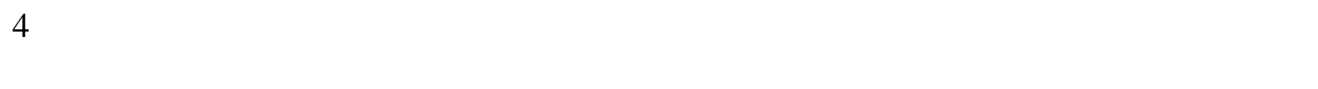 \\
\hline Position & Isometric exercise protocol including 4 exercises & Knee flexion and torso erect on same vibration platform used in LV \\
\hline Sessions & 1 & 1 \\
\hline $\begin{array}{l}\text { Sets/Time series/ } \\
\text { rest }\end{array}$ & $4 \times 1 \mathrm{~min} / 30 \mathrm{~s}$ & $4 \times 1 \mathrm{~min} / 45 \mathrm{~s}$ \\
\hline $\begin{array}{l}\text { Level of } \\
\text { evidence }\end{array}$ & II & III-1 \\
\hline
\end{tabular}




\section{Morel et al., Afr J Tradit Complement Altern Med., (2017) 14(S): 19-27 \\ https://doi.org/10.21010/ajtcam.v14i4S.3}

Kurt and Pekünlü, (2015) have evaluated handgrip, squat jump, trunk flexion and isometric leg strength tests after each intervention. They have investigated the effect of WBVE training on maximal strength, squat jump, and flexibility of well-trained combat athletes. Kurt (2015) has assessed the static SS, and DS - in four sessions of equal duration 48 hours apart in a randomized balanced order. During a 15-minute recovery after each protocol, subjects performed the stand and reach test (S\&R). He compared the effect of local vibration (LV) and WBVE on lower body flexibility and to assess whether vibration treatments were more effective than traditionally used SS and DS methods. Kurt (2015) observed that there was a similar change pattern in S\&R scores across the 15-minute recovery after each protocol $(\mathrm{p}=0.572)$, remaining significantly elevated throughout recovery. A significant main protocol effect was found for absolute change in S\&R scores relative to baseline $(p=0.015)$. These changes were statistically greater in LV than WBVE and DS. Changes in SS were not significantly different from LV, but were consistently lower than LV with almost moderate effect sizes. After LV, a greater percentage of subjects increased flexibility above the minimum detectable change compared to other protocols; while Kurt and Pekünlü, (2015) has verified significant interaction ( $\mathrm{p}=$ $0.018)$ of pre-post $\times$ intervention only for the handgrip test, indicating a significant performance increase of moderate effect (net increase of $2.48 \%, \mathrm{~d}=0.61$ ) after WBVE intervention. Squat jump, trunk flexion and isometric leg strength performances were not affected by WBVE. In all studies, synchronicus platform was used and the frequency ranged slightly from 26 up to $30 \mathrm{~Hz}$. Individuals were on the base of the platform, performing isometric exercise including four different exercises in static squat at $120^{\circ}$ knee flexion and a dynamic squat of 2 sec up and 2 sec down at a knee angle ranging from $120^{\circ}$ to $180^{\circ}$ (Kurt, 2015) or they were with the knee joint angle and torso erect on the same vibration platform used in LV (Kurt and Pekünlü, 2015). A single session was used in the two publications. The working time in the sessions varied from was the same (four 1-min bouts), but the rest time was 30 sec (Kurt, 2015) and $45 \mathrm{sec}$ (Kurt, 2015).

\section{Discussion}

Sports exhibit different responses to identical training stimulus. No specific study in the literature has investigated whether WBVE enhances performance in athletes that practice different sports. In this current review is shown the relevance of a same stimulus, the WBVE, in athletes of soccer, diver and combat sports.

Putting together all the investigations involving WBVE in soccer and combat sports, it is verified the necessity of developing studies with similar outcomes. Moreover, a standardized methodology and reporting of key variable is required between investigators to allow for a better comparison of studies.

About investigations involving WBVE and divers, it is necessary develop studies with similar outcomes and consider new studies in which the athletes would be in the same position on the base of the OVP.

The current study has several limitations that must be considered in the interpretation of the findings. Caution should be taken when generalizing the results due to the methodological variations concerning to biomechanical parameters and variability of protocols used. Although we tried to retrieve the articles involving WBVE and specific sports with selected keywords, it is not sure that all studies on this topic have been identified, including articles that were not published in English and articles published in journals that were not indexed in the PubMed and PEDRo databases. Limited number of publications with high methodological quality (RCTs) must also be considered and this fact could affect the evidence of the findings.

The use of WBVE has proven to be a safe and useful strategy to improve physical conditions of players of soccer, diver and combat sports. Nevertheless, methodological flaws and differences among protocols indicate the need of more research in this area.

\section{References}

1. Claybaugh, J. R. Lin, Y. C. (2004). Exercise and decompression sickness: a matter of intensity and timing. J Physiol. 555(Pt 3): 588.

2. Cloak, R., Nevill, A., Wyon, M. (2016). The acute effects of vibration training on balance and stability amongst soccer players. Eur J Sport Sci. 16: 20-26.

3. Cochrane, D. J. (2011). Vibration exercise: the potential benefits. Int J Sports Med. 32: 75-99.

4. Dallas, G., Paradisis, G., Kirialanis, P., Mellos, V., Argitaki, P., Smirniotou, A. (2015). The acute effects of different training loads of whole body vibration on flexibility and explosive strength of lower limbs in divers. Biol Sport. 32: 235-241.

5. Germonpre, P., Pontier, J.M., Gempp, E., Blatteau, J.E., Deneweth, S., Lafère, P., Marroni, A., Balestra, C. (2009). Pre-dive vibration effect on bubble formation after a $30-\mathrm{m}$ dive requiring a decompression stop. Aviat Space Environ Med. 80: 1044-1048.

6. Kurt, C. (2015). Alternative to traditional stretching methods for flexibility enhancement in well-trained combat athletes: local vibration versus whole-body vibration. Biol Sport. 32: 225-233.

7. Kurt, C., Pekunlu, E. (2015). Acute effect of whole body vibration on isometric strength, squat jump, and flexibility in well-trained combat athletes. Biol Sport. 32: 115-122. 
8. Lee, H. C., Chen, Y.S., Kang, B.H., Wan, F.J., Chang, L.P., Huang, K.L. (2009). Serum heat shock protein after simulated deep diving in Navy divers. Chin J Physiol. 52: 295-298.

9. Lovell, R., Midgley, A., Barrett, S., Carter, D., Small, K. (2013). Effects of different half-time strategies on second half soccer-specific speed, power and dynamic strength. Scand J Med Sci Sports. 23: 105-113.

10. Marín, P. J., Rhea, M.R. (2010). Effects of vibration training on muscle strength: a meta-analysis. J Strength Cond Res. 24: 548-556.

11. Merlin, T., Weston, A., Tooher, R. (2009). Extending an evidence hierarchy to include topics other than treatment: revising the Australian 'levels of evidence'. BMC Med Res Methodol. 9: 34.

12. Osawa, Y., Oguma, Y. (2013). Effects of vibration on flexibility: a meta-analysis. J Musculoskelet Neuronal Interact. 13: 442-453.

13. Padulo, J., Di Giminiani, R., Ibba, G., Zarrouk, N., Moalla, W., Attene, G., Migliaccio, G.M., Pizzolato, F., Bishop, D., Chamari, K. (2014). The acute effect of whole body vibration on repeated shuttle-running in young soccer players. Int J Sports Med. 35: 49-54.

14. Rauch, F., Sievanen, H., Boonen, S., Cardinale, M., Degens, H., Felsenberg, D., Roth, J., Scholnan, E., Verschueren, S., Rittweger, J. (2010). Reporting whole-body vibration intervention studies: recommendations of the International Society of Musculoskeletal and Neuronal Interactions. J Musculoskelet Neuronal Interact. 10: 193-198.

15. Rittweger, J. (2010). Vibration as an exercise modality: how it may work, and what its potential might be. Eur J Appl Physiol. 108: 877-904.

16. Rønnestad B.R., Ellefsen S. (2011). The effects of adding different whole-body vibration frequencies to preconditioning exercise on subsequent sprint performance. J Strength Cond Res. 25: 3306-3310.

17. Signorile, J. (2011). Whole body vibration, part one: what's shakin' now ? J Act Aging. 9.

18. Wyon, M., Guinan D., Hawkey, A. (2010). Whole-body vibration training increases vertical jump height in a dance population. J Strength Cond Res. 24: 866-870. 